\title{
Targeting Phosphodiesterase 4 to Block the Link between Acute Exacerbation of Chronic Obstructive Pulmonary Disease and the Metabolic Complications
}

\author{
Eric Cho \\ Spartan Health Sciences University, Vieux Fort, St. Lucia, Eastern Caribbean Island \\ Email: echombigah@gmail.com \\ Received 27 July 2015; accepted 3 November 2015; published 6 November 2015 \\ Copyright (C) 2015 by author and Scientific Research Publishing Inc. \\ This work is licensed under the Creative Commons Attribution International License (CC BY). \\ http://creativecommons.org/licenses/by/4.0/

(c) (i) Open Access

\begin{abstract}
The metabolic disorders such as obesity and diabetes are found to be more frequent in chronic obstructive pulmonary disease (COPD). The chronic systemic inflammation orchestrated by macrophages constitutes one critical pathophysiological process underlying both acute exacerbation of COPD (AECOPD) and its metabolic complications such as obesity and diabetes. The cyclic adenosine monophosphate (cAMP) signaling controlled by phosphodiesterase (PDE) 4 is a pivotal intracellular modulator for macrophages functions in immune inflammatory response underlying AECOPD as well as obesity and diabetes. Targeting PDE4/cAMP signaling has been suggested to be effective in treating AECOPD or the metabolic disorders of obesity and diabetes. It is therefore reasonable to hypothesize that the chronic systemic inflammation can be a critical link between AECOPD and the metabolic disorders and targeting the PDE4/cAMP signaling can be effective to block this link between AECOPD and the associated metabolic complications.
\end{abstract}

\section{Keywords}

COPD, Metabolic Disorders, PDE4, cAMP, Macrophages

\section{Introduction}

Chronic obstructive pulmonary disease (COPD) has been recognized as the third leading cause of death by the year 2020 [1]. COPD is a preventable and treatable disease associated with extra-pulmonary effects by definition. The major characteristics of COPD are emphysema, bronchitis, and small airways disease associated with

How to cite this paper: Cho, E. (2015) Targeting Phosphodiesterase 4 to Block the Link between Acute Exacerbation of Chronic Obstructive Pulmonary Disease and the Metabolic Complications. Journal of Biosciences and Medicines, 3, 62-67. 
pulmonary hypertension. The major pathological processes include the airway narrowing due to inflammation, mucus plugging and fibrosis, and pulmonary parenchymal destruction with subsequent airway closure. Tobacco smoke is the greatest risk factor for COPD development. Other risk factors include air pollution, occupational dusts and chemical, old age and some gene such as alpha-1-antitrypsin deficiency. Studies have showed that the metabolic disorders are found to be more frequent in COPD. Over the past couple of decades, increasing published data has demonstrated that acute exacerbation of COPD (AECOPD) presents with a clinical syndrome involving diversified extrapulmonary manifestations [2]. These extra pulmonary manifestations include predominantly the cardiovascular diseases and metabolic disorders in nature [3]. Patients with COPD and the metabolic disorders have much higher risk of morbidity and mortality due to the metabolic and cardiovascular diseases.

\section{Prevalence of the Metabolic Disorders in AECOPD}

In fact, metabolic abnormalities, such as type 2 diabetes mellitus (T2DM), obesity and the metabolic syndrome, are far more common in COPD patients than the general population [4]. In a study conducted in Madrid, among 6400 COPD patients, $20 \%$ patients had diabetes, $25 \%$ were obese, and $34 \%$ had dyslipidemia. In some other studies, the overall prevalence of obesity is $18 \%-25 \%$ and $5 \%-12 \%$ for diabetes development in subjects with mild to moderate COPD [4]. Especially, the impaired lung function has been suggested to be a strong risk factor for developing diabetes. According to the study by Mannino et al., the COPD subjects with severe reduced lung functions had a increased risk for diabetes (odds ratio of 1.5, CI: 1.1 - 1.9) [5]. In another prospective study over an 8-year period, the COPD patients had a 1.8\% relative risk for [6]. Dyslipidemia, one major component in the metabolic disorders, has also been suggested to be much higher prevalence in COPD patients (about 48.3\%) than the controls (about 31.7\%). Accordingly, statins was associated with significantly reduced death rate in COPD patients (30\%) over a prospective period of more than 2 years treatment, supporting the imperativeness to control the extra pulmonary metabolic abnormalities in COPD patients [7] [8].

It is true that the relationship between AECOPD and the development of these extra pulmonary metabolic derangements has been increasingly recognized. AECOPD together with these extra pulmonary manifestations constitutes the morbidity and mortality in COPD patients. Statistic data showed that AECOPD causes 50\% mortality at 3.6 years, with about $75 \%$ mortality at 7.7 years and $96 \%$ mortality upon 17 years in the COPD population [9]. Nevertheless, the mechanism underlying these clinical presentations remains unclear.

\section{Macrophages-Mediated Systemic Inflammation Constitutes the Link between AECOPD and the Metabolic Complications}

Systemic inflammation has been well suggested to be an important contributor for both COPD and metabolic disorders such as obesity and T2DM [10]. In COPD patients, chronic inflammation typically presented in the airways, lung tissue and pulmonary blood vessels. During COPD process, the inhalation of noxious substances such as tobacco smoke triggers the abnormal inflammatory response which causes structural changes and oxidative stress in the small airways and pulmonary parenchyma. Oxidative stress further amplified the inflammation, resulting in frequent COPD exacerbations. One of the key inflammatory cells during COPD are macrophages existing in airway lumen, lung parenchyma and broncoalveolar lavage fluid [11]. These macrophages, the major component of innate immune response, control the functions of T-lymphocytes and the production of pro-/antiinflammatory cytokines, determining AECOPD and the progression of the disease [12].

About two decades ago, the first links between chronic inflammation and obesity and insulin resistance has been identified. According to the studies by Spiegelman and his colleagues, in the pathogenesis of obesityinduced T2DM, insulin resistance plays a pivotal and causal function. Tumor necrosis factor (TNF) $\alpha$, a proinflammatory cytokine produced by the adipose tissue of obese animals, promotes insulin resistance through post-transcriptional modification (ser phosphorylation) of insulin receptor substrate 1 [13]. To initiate and control this inflammatory response cascade, the immune cells infiltrate the obese adipose tissue, with macrophages being functionally and numerically dominant. Approximately $45 \%$ - 60\% macrophages in the adipose tissue of obese mice can be found, whereas only $10 \%-15 \%$ of cells in the adipose tissue of lean mice [14]. Further, macrophages in adipose tissue have been found to present different phenotypes with contrast functions, alternatively activated activation (AMA or M2) with function to attenuate inflammatory response and classically macrophages activation (CMA or M1) with pro-inflammatory function and "crown-like" structures around dying 
adipocytes [15].

Currently, the evidence for the function of M2 phenotype in AECOPD has been limited. Nevertheless, the above data clearly suggests a critical function of macrophages in both AECOPD and the metabolic disorders of obesity and T2DM. It is therefore that macrophages-mediated systemic inflammation constitutes potentially the critical link between AECOPD and the metabolic complications. Thereby, how to modulate the macrophages function has been the critical issue in controlling AECOPD and the metabolic disorders of obesity and diabetes, the major extra pulmonary complications.

\section{PDE4/cAMP Signaling Determines the Macrophage-Mediated Inflammation Underlying AECOPD and Metabolic Disorders}

The functions of macrophages have been demonstrated to be modulated by both cell-extrinsic and -intrinsic mechanisms. The cell-extrinsic mechanisms include the circulating altered substances, such as noxious materials from tobacco smoke in AECOPD and the circulating adipokines and fatty acids in obesity. The cell-intrinsic pathways include abnormal functions of the subcellular organelles such as mitochondrial dysfunction and endoplasmic reticulum stress [16]. As for the intrinsic signaling, cyclic adenosine monophosphate (cAMP) pathway has been demonstrated to be fundamental to maintain macrophages' functions. The cAMP signaling involves the binding and activation of G-protein-coupled receptor which in turn leads to the activation of adenylate cyclases [17]. Elevated cAMP then initiates the downstream enzymatic signaling cascades. Phosphodiesterase (PDE) 4 is specific for degrading cAMP and accounts for the largest percentage of cAMP hydrolysis [18].

There are 4 genes to encode PDE4 including PDE4A, PDE4B, PDE4C and PDE4D. Each of these genes has unique cellular distribution and function. PDE4B represents the major isoform in PDE4 family in macrophages [19]. The critical function of PDE4-modulated macropahges inflammation in both AECOPD and metabolic disorders has been well suggested. The expression of PDE4 was upregualted accompanied with decreased cAMP levels in the macrophages isolated from broncoalveolar lavage fluid in patients with AECOPD as well as in adipose tissue from both obese mice and human. Generally, activation of cAMP signaling is associated with decreased M1 inflammatory response. For example, mice with PDE4B deficiency demonstrated attenuated LPSinduced inflammatory response and TNF- $\alpha$ production. In human monocytes and monocyte-derived cells, the transcription of PDE4B was activated by lipopolysaccharide (LPS) stimulation and was inhibited by interleukin (IL) 10, an important anti-inflammatory cytokine [20]. In obese mice, genetic deficiency of PDE4B resulted in lower body weights, reduced fat pad weights and smaller adipocytes accompanied with decreased TNF- $\alpha$ expression [21]. In the study by Luan B et al., activated cAMP signaling via leptin inhibited NF- $\kappa$ B activity, a well known pro-inflammatory transcription factor, and suppressed the macrophages inflammation mediated by cAMP-PKA signaling and the downstream histone deacetylase 4 activities [22]. Therefore, these data indicates that PDE4/cAMP signaling can be the pivotal link in determining the macrophage-mediated inflammation underlying both AECOPD and the metabolic complications.

\section{Targeting PDE4/cAMP Signaling to Block the Link between COPD and the Associated Metabolic Complications}

Up to date, increasing evidence has also revealed the therapeutic potentiality of Roflumilast in treating T2DM. It was reported that Roflumilast and Roflumilast-N-oxide abolished the deterioration of blood glucose and hemoglobin A1c (HbA1c) concomitant with preservation of pancreatic islet morphology based on Lep ${ }^{d b}$ mice model (mice homozygous for the diabetes spontaneous mutation) [23]. In another study, resveratrol, a polyphenol in red wine, exerted its effects through competitive inhibition of cAMP-degrading PDE, especially PDE4, presented with multiple metabolic benefits in high-fat diet-induced obese diabetic mice, including preventing diet-induced obesity and improving mitochondrial function and glucose tolerance [24] [25]. In a prospective, randomized, double-blind and placebo-controlled multicenter study based on outpatients with T2DM, Roflumilast decreased both fasting and postprandial glucose levels after 12 weeks treatment [26]. In another study, in a prospective randomized open-label study in 36 obese women with polycystic ovary syndrome, a disorder associated with insulin resistance and obesity, Roflumilast treatment over 12-weeks reduced body weight, primarily due to a loss of fat mass [27]. Based on the above evidence, it is therefore reasonable to hypothesize that the effects induced by PDE4i can be mostly like due to the remodeling of macrophages inflammation.

For PDE4i in COPD, a bulk of strong evidence has been existed to suggest that cAMP signaling activation via 


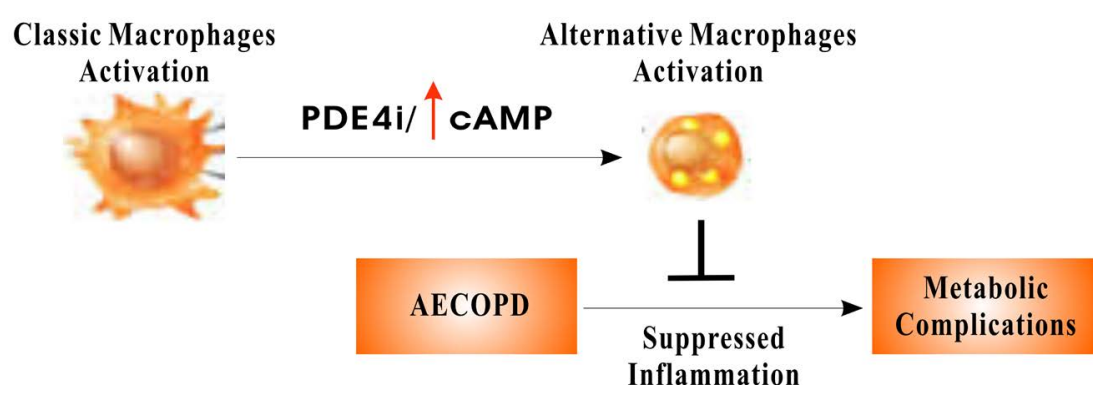

Figure 1. Targeting PDE4 4 to block the link between AECOPD and the metabolic complications. Patients of AECOPD present with classic macrophages activation with enhanced systemic immune inflammatory response. PDE4i increases cAMP levels, which promote the alternative macrophages activation with strong anti-inflammatory characteristics. Thereby, PDE4i blocks the link between AECOPD and its metabolic complications. AECOPD, Acute Exacerbation of Chronic Obstructive Pulmonary Disease; PDE, phosphodiesterase. PDE4i, PDE4 inhibitor; cAMP, cyclic adenosine monophosphate.

PDE4i in structural and inflammatory macrophages provides anti-inflammatory response in both mice models and COPD patients [28]. Roflumilast is the first PDE4 inhibitor to be approved for treating COPD patients with exacerbation. In human lung parenchymal explants, Roflumilast and its active metabolite of Roflumilast$\mathrm{N}$-oxide concentration-dependently reduced the release of TNF- $\alpha$ and the pro-inflammatory chemokines from LPS-stimulated human lung explants [29]. Further, Roflumilast suppressed the proliferation and cytokines release from macrophages and T lymphocytes which involves inhibiting the activation of NF- $\kappa \mathrm{B}$. Roflumilast has been progressed in late Phase III clinical trials with bioavailability of $79 \%$ and once daily due to its favorable pharmacokinetics [30]. In addition, Roflumilast is safe and well tolerated without significant interactions with other COPD drugs. The major side effects of Roflumilast, although rare, include some gastrointestinal responses such as dyspepsia, gastritis and vomiting.

\section{Conclusion and Future Directions}

The above results provide strong evidence that PDE4i (Roflumilast) is highly efficient in treating both COPD and metabolic disorders such as obesity and diabetes. Although the causal relationship between AECOPD and the metabolic complications has yet to be demonstrated, systemic inflammation can be one of the critical underlying mechanisms underlying these disorders. Given the growing worldwide problem of COPD in relation to its related complications and comorbidities, strategies for prevention and treatment are essential. It is therefore reasonable to hypothesize that PDE4i (Roflumilast) can be effective in suppressing the systemic inflammation, and then blocking the link between AECOPD and the metabolic complications (Figure 1).

\section{Conflict of Interests}

None.

\section{References}

[1] Pauwels, R.A. and Rabe, K.F. (2004) Burden and Clinical Features of Chronic Obstructive Pulmonary Disease (COPD). Lancet, 364, 613-620. http://dx.doi.org/10.1016/S0140-6736(04)16855-4

[2] Brody, H. (2012) Chronic Obstructive Pulmonary Disease. Nature, 489, S1. http://dx.doi.org/10.1038/489S1a

[3] MacIntyre, N. and Huang, Y.C. (2008) Acute Exacerbations and Respiratory Failure in Chronic Obstructive Pulmonary Disease. Proceedings of the American Thoracic Society, 5, 530-535. http://dx.doi.org/10.1513/pats.200707-088ET

[4] Garcia-Olmos, L., Alberquilla, A., Ayala, V., et al. (2013) Comorbidity in Patients with Chronic Obstructive Pulmonary Disease in Family Practice: A Cross Sectional Study. BMC Family Practice, 14, 11. http://dx.doi.org/10.1186/1471-2296-14-11

[5] Celli, B.R., Thomas, N.E., Anderson, J.A., et al. (2008) Effect of Pharmacotherapy on Rate of Decline of Lung Function in Chronic Obstructive Pulmonary Disease: Results from the TORCH Study. American Journal of Respiratory and 
Critical Care Medicine, 178, 332-338. http://dx.doi.org/10.1164/rccm.200712-1869OC

[6] Rana, J.S., Mittleman, M.A., Sheikh, J., et al. (2004) Chronic Obstructive Pulmonary Disease, Asthma, and Risk of Type 2 Diabetes in Women. Diabetes Care, 27, 2478-2484. http://dx.doi.org/10.2337/diacare.27.10.2478

[7] Neukamm, A., Hoiseth, A.D., Einvik, G., et al. (2015) Rosuvastatin Treatment in Stable Chronic Obstructive Pulmonary Disease (RODEO): A Randomized Controlled Trial. Journal of Internal Medicine, 278, 59-67. http://dx.doi.org/10.1111/joim.12337

[8] Horita, N., Miyazawa, N., Kojima, R., et al. (2014) Statins Reduce All-Cause Mortality in Chronic Obstructive Pulmonary Disease: A Systematic Review and Meta-Analysis of Observational Studies. Respiratory Research, 15, 80.

[9] Suissa, S., Dell’Aniello, S. and Ernst, P. (2012) Long-Term Natural History of Chronic Obstructive Pulmonary Disease: Severe Exacerbations and Mortality. Thorax, 67, 957-963. http://dx.doi.org/10.1136/thoraxjnl-2011-201518

[10] Chang, C., Zhu, H., Shen, N., et al. (2015) Bacterial Infection, Airway and Systemic Inflammation and Clinical Outcomes before and after Treatment of AECOPD, a Longitudinal and Cross-Sectional Study. Copd, 12, 19-30. http://dx.doi.org/10.3109/15412555.2014.898043

[11] Boorsma, C.E., Draijer, C. and Melgert, B.N. (2013) Macrophage Heterogeneity in Respiratory Diseases. Mediators of Inflammation, 2013, Article ID: 769214. http://dx.doi.org/10.1155/2013/769214

[12] Barnes, P.J. (2013) New Anti-Inflammatory Targets for Chronic Obstructive Pulmonary Disease. Nature Reviews Drug Discovery, 12, 543-559. http://dx.doi.org/10.1038/nrd4025

[13] Chawla, A., Nguyen, K.D. and Goh, Y.P. (2011) Macrophage-Mediated Inflammation in Metabolic Disease. Nature Reviews Immunology, 11, 738-749. http://dx.doi.org/10.1038/nri3071

[14] Xu, H.Y., Barnes, G.T., Yang, Q., Tan, G., Yang, D., Chou, C.J., et al. (2003) Chronic Inflammation in Fat Plays a Crucial Role in the Development of Obesity-Related Insulin Resistance. The Journal of Clinical Investigation, 112, 1821-1830. http://dx.doi.org/10.1172/JCI200319451

[15] Lumeng, C.N., Bodzin, J.L. and Saltiel, A.R. (2007) Obesity Induces a Phenotypic Switch in Adipose Tissue Macrophage Polarization. The Journal of Clinical Investigation, 117, 175-184. http://dx.doi.org/10.1172/JCI29881

[16] Zhao, L., Zhong, S., Qu, H.Y., Xie, Y.X., Cao, Z.N., Li, Q., et al. (2015) Chronic Inflammation Aggravates Metabolic Disorders of Hepatic Fatty Acids in High-Fat Diet-Induced Obese Mice. Scientific Reports, 5, Article No.: 10222. http://dx.doi.org/10.1038/srep10222

[17] Pierce, K.L., Premont, R.T. and Lefkowitz, R.J. (2002) Seven-Transmembrane Receptors. Nature Reviews Molecular Cell Biology, 3, 639-650. http://dx.doi.org/10.1038/nrm908

[18] Sprenger, J.U., Perera, R.K., Steinbrecher, J.H., Lehnart, S.E., Maier, L.S., Hasenfuss, G. and Nikolaev, V.O. (2015) In Vivo Model with Targeted cAMP Biosensor Reveals Changes in Receptor-Microdomain Communication in Cardiac Disease. Nature Communications, 6, Article No.: 6965. http://dx.doi.org/10.1038/ncomms7965

[19] Jin, S.L., Lan, L., Zoudilova, M. and Conti, M. (2005) Specific Role of Phosphodiesterase 4B in LipopolysaccharideInduced Signaling in Mouse Macrophages. Journal of Immunology, 175, 1523-1531. http://dx.doi.org/10.4049/jimmunol.175.3.1523

[20] Jin, S.L. and Conti, M. (2002) Induction of the Cyclic Nucleotide Phosphodiesterase PDE4B Is Essential for LPS-Activated TNF-Alpha Responses. Proceedings of the National Academy of Sciences of the United States of America, 99, 7628-7633. http://dx.doi.org/10.1073/pnas.122041599

[21] Zhang, R., Maratos-Flier, E. and Flier, J.S. (2009) Reduced Adiposity and High-Fat Diet-Induced Adipose Inflammation in Mice Deficient for Phosphodiesterase 4B. Endocrinology, 150, 3076-3082. http://dx.doi.org/10.1210/en.2009-0108

[22] Luan, B., Goodarzi, M.O., Phillips, N.G., Guo, X.Q., Chen, Y.-D.I., Yao, J., et al. (2014) Leptin-Mediated Increases in Catecholamine Signaling Reduce Adipose Tissue Inflammation via Activation of Macrophage HDAC4. Cell Metabolism, 19, 1058-1065. http://dx.doi.org/10.1016/j.cmet.2014.03.024

[23] Vollert, S., Kaessner, N., Heuser, A., Hanauer, G., Dieckmann, A., Knaack, D., et al. (2012) The Glucose-Lowering Effects of the PDE4 Inhibitors Roflumilast and Roflumilast- $N$-Oxide in $\mathrm{db} / \mathrm{db}$ Mice. Diabetologia, 55, 2779-2788. http://dx.doi.org/10.1007/s00125-012-2632-z

[24] Yan, L., Park, J.Y., Dillinger, J.G., De Lorenzo, M.S., Yuan, C.J., Lai, L., et al. (2012) Common Mechanisms for Calorie Restriction and Adenylyl Cyclase Type 5 Knockout Models of Longevity. Aging Cell, 11, 1110-1120. http://dx.doi.org/10.1111/acel.12013

[25] Jeon, B.T., Jeong, E.A., Shin, H.J., Lee, Y., Lee, D.H., Kim, H.J., et al. (2012) Resveratrol Attenuates Obesity-Associated Peripheral and Central Inflammation and Improves Memory Deficit in Mice Fed a High-Fat Diet. Diabetes, 61, 1444-1454. http://dx.doi.org/10.2337/db11-1498

[26] Wouters, E.F., Bredenbroker, D., Teichmann, P., Brose, M., Rabe, K.F., Fabbri, L.M. and Göke, B. (2012) Effect of 
the Phosphodiesterase 4 Inhibitor Roflumilast on Glucose Metabolism in Patients with Treatment-Naive, Newly Diagnosed Type 2 Diabetes Mellitus. The Journal of Clinical Endocrinology and Metabolism, 97, E1720-E1725. http://dx.doi.org/10.1210/jc.2011-2886

[27] Jensterle, M., Kocjan, T. and Janez, A. (2014) Phosphodiesterase 4 Inhibition as a Potential New Therapeutic Target in Obese Women with Polycystic Ovary Syndrome. The Journal of Clinical Endocrinology and Metabolism, 99, E1476E1481. http://dx.doi.org/10.1210/jc.2014-1430

[28] Fabbri, L.M., Beghe, B., Yasothan, U. and Kirkpatrick, P. (2010) Roflumilast. Nature Reviews Drug Discovery, 9, 761762. http://dx.doi.org/10.1038/nrd3276

[29] Buenestado, A., Chaumais, M.C., Grassin-Delyle, S., Risse, P.-A., Naline, E., Longchampt, E., et al. (2013) Roflumilast Inhibits Lipopolysaccharide-Induced Tumor Necrosis Factor-Alpha and Chemokine Production by Human Lung Parenchyma. PLoS ONE, 8, e74640. http://dx.doi.org/10.1371/journal.pone.0074640

[30] Martinez, F.J., Calverley, P.M., Goehring, U.M., Brose, M., Fabbri, L.M. and Rabe, K.F. (2015) Effect of Roflumilast on Exacerbations in Patients with Severe Chronic Obstructive Pulmonary Disease Uncontrolled by Combination Therapy (REACT): A Multicentre Randomised Controlled Trial. The Lancet, 385, 857-866. http://dx.doi.org/10.1016/S0140-6736(14)62410-7

\author{
Abbreviations \\ COPD: chronic obstructive pulmonary disease \\ AECOPD: acute exacerbation of COPD \\ PDE: phosphodiesterase \\ cAMP: cyclic adenosine monophosphate \\ T2DM: type 2 diabetes mellitus \\ TNF- $\alpha$ : Tumor necrosis factor $-\alpha$ \\ AMA or M2: alternatively activated activation \\ CMA or M1: classically macrophages activation \\ LPS: lipopolysaccharide \\ IL-10: interleukin-10 \\ HbA1c: hemoglobin A1c
}

